\title{
Dynamic Fault Classification AND Location in DisTRIBUTION NETWORKS
}

\author{
Abdelhakim BOURICHA*, Tahar BOUTHIBA, Samira SEGHIR, \\ Rebiha BOUKHARI
}

Power System Optimization Laboratory(LORE) University of Sciences and Technology of Oran Mohammed Boudiaf, USTO B.P. 1505 El-Mnaouar, Oran 31000 - Algeria

*Corresponding Author: Abdelhakim BOURICHA (email: abdelhakim.bouricha@univ-usto.dz) (Received: 12-March-2018; accepted: 04-September-2018; published: 31-October-2018)

DOI: http://dx.doi.org/10.25073/jaec.201823.114

\begin{abstract}
This paper presents a method for detecting, classifying and localizing faults in $M V$ distribution networks. This method is based on only two samples of current or voltage signals. The fault detection, faultclassification and fault localization are based on the maximum value of current and voltage as a function of time. A study is presented in this work to evaluate the proposed method.A comparative study between current and voltage method detection has been done to determine which is the fastest. In addition, the classification and localization of faults were made by the same method using two samples signal. Simulation with results have been obtained by using MATLAB / Simulink software. Results are reported and conclusions are drown.
\end{abstract}

\section{Keywords}

Distribution Network, Fault Detection, Fault Classification, Fault Localization.

\section{INTRODUCTION}

Electric power systems have developed rapidly in recent years and these systems have become important in all branches of the modern econ- omy. With the growth of world population, and development in all areas, the demand for electric power is growing rapidly.

Medium-Voltage electrical power distribution lines are an essential part of an electrical power grid that must ensure the continuity of power supply to Medium Voltage (MV) and Low Voltage (LV) consumers. That is not always the case, These lines experience faults which are caused by storms, lightning, snow, freezing rain, insulation breakdown and, short circuits caused by birds and other external objects [1. These faults must be detected, classified and localized quickly and correctly so that our system remains stable.

When a fault occurs in a distribution networks, the fault current is always greater than the rated load current and the fault voltage will be smaller than the nominal network voltage. The detection and localization of faults in electrical networks plays an important role in the correct operation of protective relays.

Fault detection and localization conventional methods for distribution lines are broadly classified as impedance based method which uses the steady state fundamental components of voltage and current values [2]-6]. Wavelet method which is based on low pass filters and high pass filters 77-9, and knowledge based method which uses artificial neural network and/or pattern recognition techniques [10]-12]. 
Digital relays that use the wavelet method and methods based on artificial neural networks for detecting and locating faults have a weakness because they have been designed for specific networks unlike the digital relay based on conventional algorithms that are designed on the basis of current or voltage amplitude measurements

Increase of current magnitude or decrease of voltage magnitude could be considered as a measure to detect andclassifya system in fault. The measure of reactance or impedance of the line is considered to locate the fault.

In [13] the authors use two methods to localise the fault in transmission line. The first method is based on the first and second derivative of the circuit equation and the second method is based on the integral of the circuit equation.

In this paper, an algorithm is proposed to detect, classify and locate faults on distribution network as a function of time. The method is based only on two samples of signal current or voltage.

\section{USED METHOD}

For the detection of electrical faults in any network there are several methods. Most methods use the maximum values of the voltage or current $\left(\mathrm{V}_{\max }, \mathrm{I}_{\max }\right)$ comparing them to a threshold value, in this paper we will use a new method based on two samples which is as follows:

The equation of the voltage is as follows:

$$
v=V_{\max } * \sin \left(w_{0} * t\right)
$$

We have the voltage at the moment $k$ :

$$
v_{k}=V_{\max } * \sin \left(w_{0} * t_{k}\right)
$$

The voltage at the moment $k+1$ :

$$
\begin{gathered}
v_{k}=V_{\max } * \sin \left(w_{0} * t_{k}\right) \\
v_{k+1}=V_{\max } * \sin \left(w_{0} *\left(t_{k}+\Delta t\right)\right) \\
v_{k+1}=V_{\max } * \sin \left(\left(w_{0} * t_{k}\right)+\left(w_{0} * \Delta t\right)\right) \\
\Delta t=t_{k+1}-t_{k}=0.001 \mathrm{sec} .
\end{gathered}
$$

We know that:

$$
\sin (A+B)=\sin A * \cos B+\cos A * \sin B
$$

Therefore, Eq. (5) becomes:

$$
\begin{aligned}
v_{k+1} & =V_{\max } * \sin \left(w_{0} * t_{k}\right) * \cos \left(w_{0} * \Delta t\right) \\
& +V_{\max } * \cos \left(w_{0} * t_{k}\right) * \sin \left(w_{0} * \Delta t\right) .
\end{aligned}
$$

We replace Eq. (2) in Eq. (7) and we get:

$$
\begin{aligned}
v_{k+1} & =v_{k} * \cos \left(w_{0} * \Delta t\right) \\
& +V_{\max } * \cos \left(w_{0} * t_{k}\right) * \sin \left(w_{0} * \Delta t\right)
\end{aligned}
$$

So

$V_{\max } * \cos \left(w_{0} * t_{k}\right)=\frac{v_{k+1}-v_{k} * \cos \left(w_{0} * \Delta t\right)}{\sin \left(w_{0} * \Delta t\right)}$

With (Eq. 22) $)^{2}+$ (Eq. (9) $)^{2}$, we give:

$$
\begin{aligned}
& V_{\max k}= \\
& \sqrt{\frac{v_{k}^{2}+v_{k+1}^{2}-2 * v_{k} * v_{k+1} * \cos \left(w_{0} * \Delta t\right)}{\left(\sin \left(w_{0} * \Delta t\right)\right)^{2}}}
\end{aligned}
$$

We do the same thing for the current " $i$ ", the result is:

$$
\begin{aligned}
& I_{\max _{k}}= \\
& \sqrt{\frac{i_{k}^{2}+i_{k+1}^{2}-2 * i_{k} * i_{k+1} * \cos \left(w_{0} * \Delta t\right)}{\left(\sin \left(w_{0} * \Delta t\right)\right)^{2}}}
\end{aligned}
$$

The current can be written as:

$$
i_{k}=I_{\max } * \sin \left(w_{0} * t_{k}+\theta_{k}\right)
$$

$$
\begin{aligned}
i_{k} & =I_{\max } * \sin \left(w_{0} * t_{k}\right) * \cos \theta_{k} \\
& +I_{\max } * \cos \left(w_{0} * t_{k}\right) * \sin \left(\theta_{k}\right)
\end{aligned}
$$

$$
\begin{gathered}
i_{k+1}=I_{\max } * \sin \left(w_{0} * t_{k+1}+\theta_{k}\right) \\
i_{k+1}=I_{\max } * \sin \left(w_{0} *\left(t_{k}+\Delta t\right)+\theta_{k}\right) \\
i_{k+1}= \\
I_{\max } *\left[\begin{array}{l}
\sin \left(w_{0} * t_{k}\right) * \cos \left(w_{0} * \Delta t\right) \\
+\cos \left(w_{0} * t_{k}\right) * \sin \left(w_{0} * \Delta t\right)
\end{array}\right] * \cos \left(\theta_{k}\right) \\
+I_{\max } *\left[\begin{array}{l}
\cos \left(w_{0} * t_{k}\right) * \cos \left(w_{0} * \Delta t\right) \\
-\sin \left(w_{0} * t_{k}\right) * \sin \left(w_{0} * \Delta t\right)
\end{array}\right] * \sin \left(\theta_{k}\right)
\end{gathered}
$$


From Eq. (2) we have:

$$
\sin \left(w_{0} * t_{k}\right)=\frac{v_{k}}{V_{\max }}
$$

From Eq. (7) we have:

$$
\cos \left(w_{0} * t_{k}\right)=\frac{v_{k+1}-v_{k} * \cos \left(w_{0} * \Delta t\right)}{V_{\max } * \sin \left(w_{0} * \Delta t\right)}
$$

Using Eq. (13) and Eq. (16) we can obtaining the expression of $\theta_{k}$. Using Eq. (17) and Eq. (18), we obtain the final value of $\theta_{k}$ :

$$
\theta_{k}=-\cos ^{-1}\left(\frac{E_{1}}{E_{2}}\right)
$$

where

$$
\begin{aligned}
& E_{1}=i_{k} * v_{k}+i_{k+1} * v_{k+1} \\
& \quad-\left(i_{k} * v_{k+1}+i_{k+1} * v_{k}\right) * \cos \left(w_{0} * \Delta t\right) \\
& E_{2}=I_{\max k} * V_{\max k} *\left(\sin \left(w_{0} * \Delta t\right)\right)^{2} .
\end{aligned}
$$

Using Eq. (2) and Eq. (12), the fault impedance $Z_{k}$ can be determined as:

$$
\begin{gathered}
Z_{k}=\frac{v_{k}}{i_{k}}=\frac{V_{\max k} * \sin \left(w_{0} * t_{k}\right)}{I_{\max k} * \sin \left(w_{0} * t_{k}+\theta_{k}\right)} \\
Z_{k}=\frac{V_{\max _{k}}}{I_{\max _{k}}} e^{-j \theta_{k}}
\end{gathered}
$$

We note:

$$
\theta_{z_{k}}=-\theta_{k}
$$

To localize the fault, the fault impedance $Z_{k}$ can be determined by:

$$
Z_{k}=\frac{V_{\max k}}{I_{\max k}} *\left(\cos \theta_{z_{k}}+j * \sin \theta_{z_{k}}\right)
$$

With:

$$
\theta_{z_{k}}=\cos ^{-1}\left(\frac{E_{3}}{E_{4}}\right)
$$

where

$$
\begin{gathered}
E_{3}=i_{k} * v_{k}+i_{k+1} * v_{k+1} \\
-\left(i_{k} * v_{k+1}+i_{k+1} * v_{k}\right) * \cos \left(w_{0} * \Delta t\right) \\
E_{4}=I \max _{k} * V \max _{k} *\left(\sin \left(w_{0} * \Delta t\right)\right)^{2} \\
Z_{k}=R_{k}+j X_{k} \\
R_{k}=\frac{V_{\max k}}{I_{\max _{k}}} *\left(\cos \theta_{z_{k}}\right) \\
X_{k}=\frac{V_{\max _{k}}}{I_{\max _{k}}} *\left(\sin \theta_{z_{k}}\right)
\end{gathered}
$$

\section{1. $\quad$ Fault detection and classification}

Fig. 1 presents the flowchart of the method to detect and classify the fault.

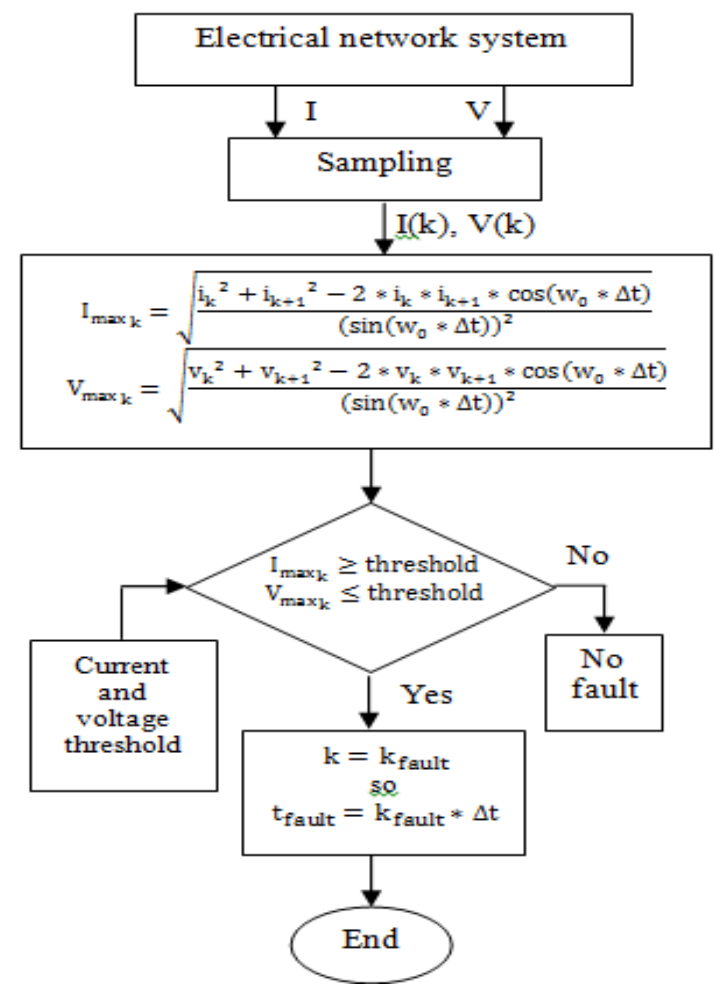

Fig. 1: The flowchart of the methodfor fault detection and classification.

\subsection{Fault localization}

The apparent positive-sequence fault impedance measured is proportional to the fault distance, which can be estimate for each fault type [14, 15. as shown in Table 1.

Where

$a, b$ and $c$ indicates faulty phases.

$g$ indicates ground fault.

$V_{a}, V_{b}$ and $V_{c}$ indicate voltage phasors.

$I_{a}, I_{b}$ and $I_{c}$ indicate current phasors.

$$
k=\frac{Z_{O L}-Z_{d L}}{3 Z_{d L}}
$$


$Z_{O L}$ is the zero-sequence line impedance.

$Z_{d L}$ is the positive-sequence line impedance.

$I_{R}$ is the residual current $\left(3 I_{0}\right)$.

$I_{0}$ is the zero- sequence current.

The fault location $(m)$ can be determined by using impedance $Z_{k}$ or the reactance $X_{k}$. Using the reactance, the fault location $(m)$ is:

$$
m=\frac{X_{k}}{X_{d}}
$$

$X_{d}$ is the positive sequence line reactance $(\Omega / \mathrm{km})$.

\section{POWER SYSTEM MODEL}

Fig. 2 shows the block Simulink of our $25 \mathrm{kV}, 50$ $\mathrm{Hz}$ network under the software MATLAB. The

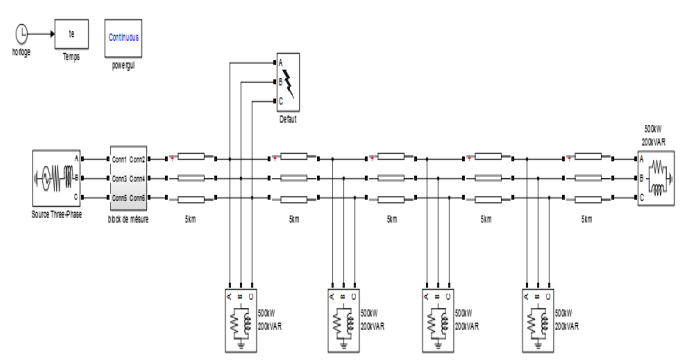

Fig. 2: Power system model.

distribution line parameters are as follows:

Positive Sequence Resistance: $R_{d}=0.2236$ $\Omega / \mathrm{km}$.

Zero Sequence Resistance: $R_{0}=0.368 \Omega / \mathrm{km}$.

Positive Sequence Inductance: $L_{d}=1.11$ $\mathrm{mH} / \mathrm{km}$.

Zero Sequence Inductance: $L_{0}=5.05 \mathrm{mH} / \mathrm{km}$.

Positive Sequence Capacitance: $C_{d}=11.13$ $\mathrm{nF} / \mathrm{km}$.

Zero Sequence Capacitance: $C_{0}=5 \mathrm{nF} / \mathrm{km}$.

The line is divided into 5 parts of $5 \mathrm{~km}$; at the end of each part, we have a load.

All loads have an active power of $500 \mathrm{~kW}$ and a reactive power of 200 kvar.

Fig. 3 shows the steps performed by the digital relay for fault detection, classification and localization.

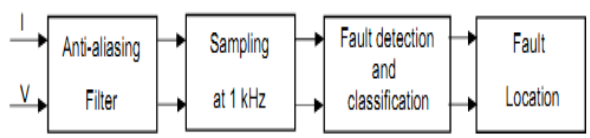

Fig. 3: The steps performed by the digital relay for fault detection, classification and localization.

The currents and voltage signals are filtered using the antialiasing filter (Butterworth lowpass) and sampled at $1 \mathrm{kHz}$.

\section{SIMULATIONSAND RESULTS}

When a fault appears in distribution line, the maximum value of current increases and the maximum value of voltage decreases. By comparing with a threshold at each sample " $k$ " we can detect and classify the fault.

\subsection{Fault detection}

To detect fault in distribution line, we can use the maximum value of the current or voltage signal.

Using the network illustrated in Fig. 2 a single-phase to ground fault (a-g) was applied at the instant $60 \mathrm{~ms}$ with a distance of $5 \mathrm{~km}$ using neutral regime connected directly to ground and a zero-fault resistance $R_{f}$ ault $=0 \Omega$. The fault is detected by both maximum voltage and currentvalues.

Fig. 4 shows the current signal, the maximum current value and the output fault detector signal in function of time.

Fig. 5 shows the voltage signal, the maximum voltage value and the output fault detector signal in function of time.

In Fig. 4(a) the black dots represent the maximum current value calculated at each instant. In Fig. 4(b) we can see, the first dot that is dif- 
Table 1. Single fault impedance equation for negligible fault resistance.

\begin{tabular}{|c|c|}
\hline Fault type & Fault impedance $Z_{k}$ \\
\hline a-g & $\frac{V_{a}}{\left(I_{a}+k I_{R}\right)}$ \\
\hline b-g & $\frac{V_{b}}{\left(I_{b}+k I_{R}\right)}$ \\
\hline c-g & $\frac{V_{c}}{\left(I_{c}+k I_{R}\right)}$ \\
\hline a-b or a-b-g & $\frac{V_{a}-V_{b}}{\left(I_{a}-I_{b}\right)}$ \\
\hline b-c or b-c-g & $\frac{V_{b}-V_{c}}{\left(I_{b}-I_{c}\right)}$ \\
\hline c-a or c-a-g & $\frac{V_{c}-V_{a}}{\left(I_{c}-I_{a}\right)}$ \\
\hline a-b-c or a-b-c-g & $\frac{V_{a}-V_{b}}{\left(I_{a}-I_{b}\right)}$ or $\frac{V_{b}-V_{c}}{\left(I_{b}-I_{c}\right)}$ or $\frac{V_{c}-V_{a}}{\left(I_{c}-I_{a}\right)}$ \\
\hline
\end{tabular}

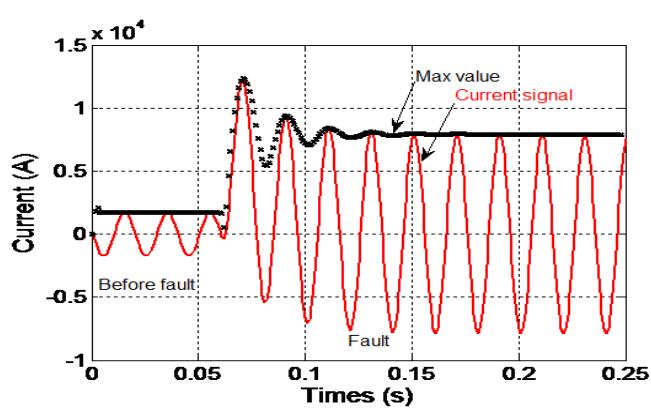

(a)

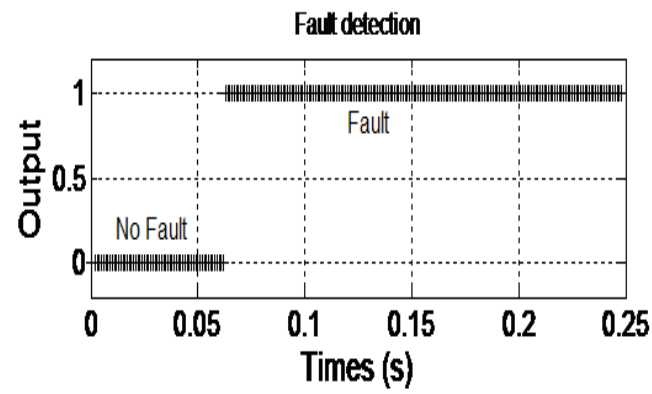

(b)

Fig. 4: Fault detector output using the maximum current value.

ferent from zero is at the instant 0.062 sec. (62 $\mathrm{ms})$. Therefore, the fault is detected $2 \mathrm{~ms}$ late.

In Fig. 5 (a) the black dots represent the maximum voltage value calculated at each instant. In Fig. 5 (b) we can see, the first dot that is different from zero is at the instant $0.061 \mathrm{sec}$. (61 ms). Therefore, the fault is detected $1 \mathrm{~ms}$ behind.

Therefore it is concluded that the detection

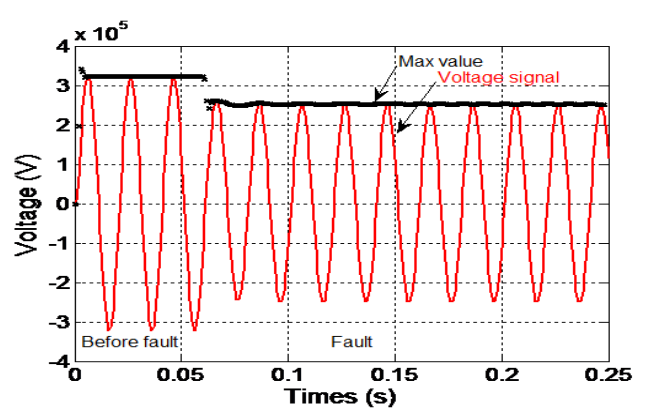

(a)

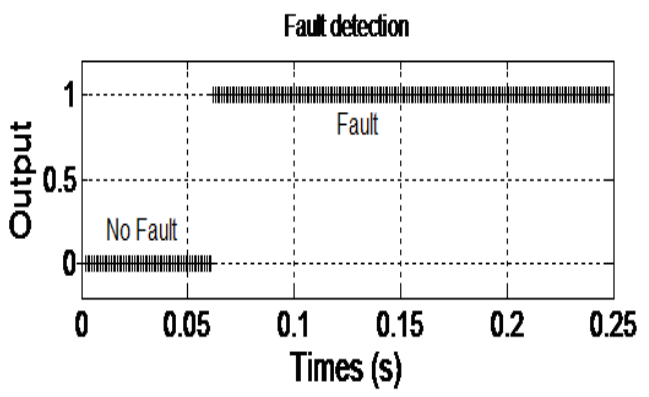

(b)

Fig. 5: Fault detector output using the maximum voltage value.

by the developed method using the maximum voltage value is faster than the detection by the maximum current value.

\subsection{Fault classification}

To classify the fault by the proposed method, the maximum voltage values are used and the 
same steps as the detection for each phase are followed. To classify the ground fault, we use the zero sequence voltage signal.

We programmed a fault classifier algorithm and we created several types of faults. The results are as follows:

Fig. 6 represents the fault classifier output as a function of time for a single-phase to ground fault (a-g).

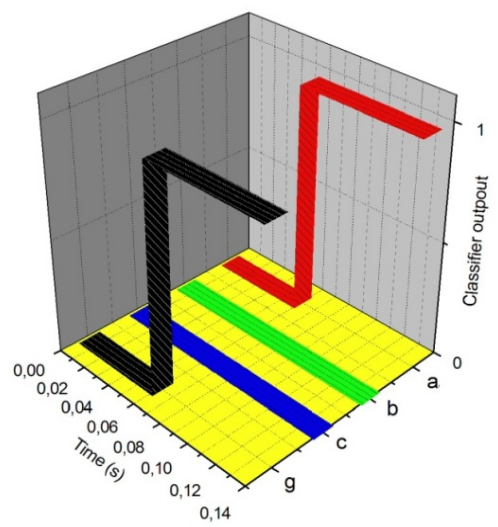

Fig. 6: Fault classifier output for the single-phase to ground fault in the phase "a".

The fault classifier indicates that the phases "b" and "c" are always zero, which implies that it is a single-phase fault (a-g). The fault is classified on phase "a" at time $0.062 \mathrm{sec}$. and on the ground at time $0.063 \mathrm{sec}$, so the fault classification time is equal to $0.063 \mathrm{sec}$, it's late by $3 \mathrm{~ms}$.

Fig. 7 represents the fault classifier output as a function of time for a double-phase fault without ground $(\mathrm{a}-\mathrm{b})$.

The fault classifier indicates that the phase "c" and the ground are always zero, which implies that it is a double-phasefault ( $a-b)$. The fault is classified on phase "a" at time $0.061 \mathrm{sec}$. and on phase "b" at time 0.062 sec. so the fault classification time is equal to $0.062 \mathrm{sec}$, it's late by $2 \mathrm{~ms}$.

Fig. 8 represents the fault classifier output as a function of time for a double-phase fault withground (a-c-g).

The fault classifier indicates that phase " $b$ " is always zero, which implies that it is a double-

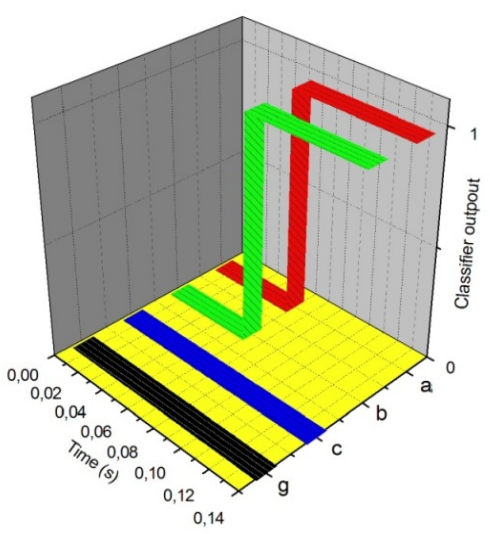

Fig. 7: Fault classifier output for the single-phase to ground fault in the phase "a".

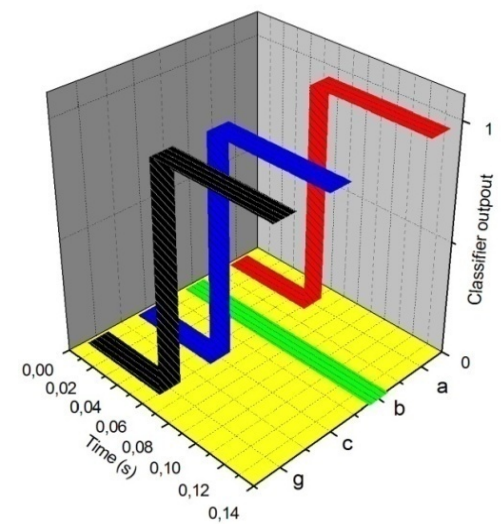

Fig. 8: Fault classifier output forthe double-phases fault with ground in the phases 'a', 'c' and the ground.

phaseto ground fault (a-c-g). The fault is classified on phase "a" at time $0.063 \mathrm{sec}$. and on phase " $c$ " and the ground at time 0.062 sec. so the fault classification time is equal to $0.063 \mathrm{sec}$, it's late by $3 \mathrm{~ms}$.

Fig. 9 represents the fault classifier output as a function of time for a three-phase fault (a-b-c).

The fault classifier indicates that the phases "a", "b" and "c" vary from "0" to "1" so we can conclude that it is a three-phase fault. The fault is classified on the phase " $b$ " at the instant 0.061 sec. and the phases "a" and "c" at the instants $0.062 \mathrm{sec}$. so the fault classification time is equal 


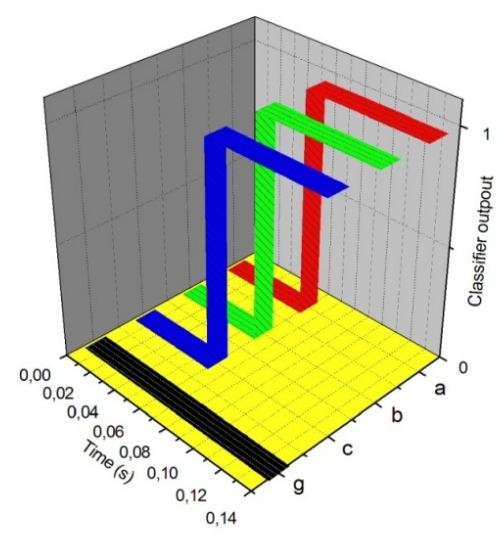

Fig. 9: Fault classifier output forthe three-phase fault'a', 'b', and 'c'.

to $0.062 \mathrm{sec}$, it's late by $2 \mathrm{~ms}$.

According to the tests studied we note that faults without ground are classified faster than faults with ground.

\subsection{Fault localization}

The fault is supposed appears at the end of each section that is to say at $5 \mathrm{~km}, 10 \mathrm{~km}, 15 \mathrm{~km}$ and $20 \mathrm{~km}$ of the distribution line.

Fig. 10 shows the fault location as a function of time using the reactance for single-phase to ground.

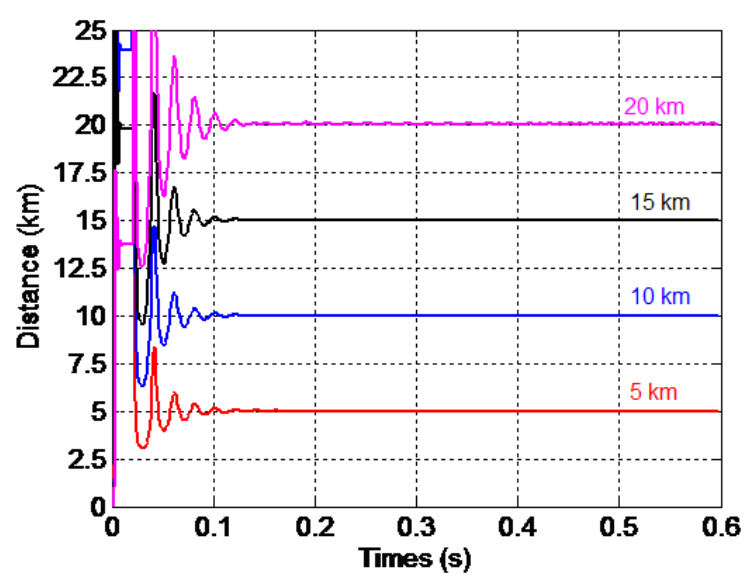

Fig. 10: Fault location as function of time using the reactance for single-phase to ground.
From Fig. 10 we can see a stability in the response and the distance is detected rapidly, it is clear that the final value of the fault locator is the same value of the supposed fault distance.

Figure 11 shows the fault location as a function of time using the reactance for double-phase fault with ground.

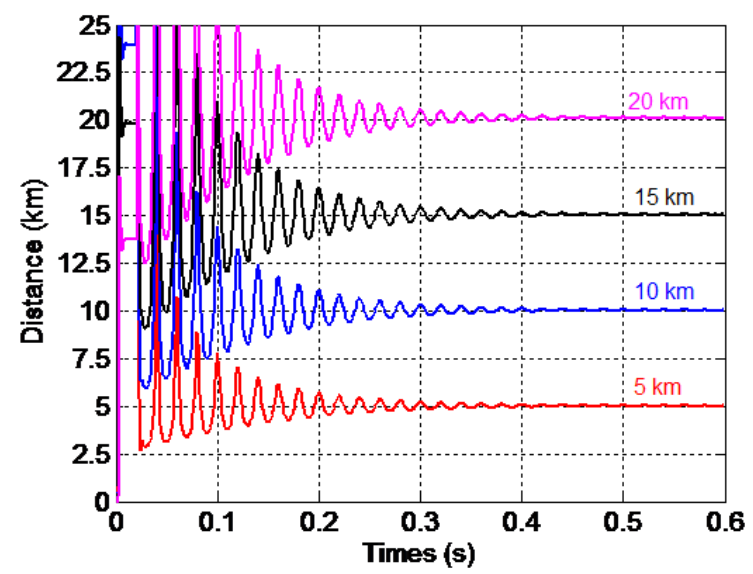

Fig. 11: Fault location as function of time using the reactance for double-phase fault with ground.

Figure 12 shows the fault location as a function of time using the reactance for double-phase fault without ground.

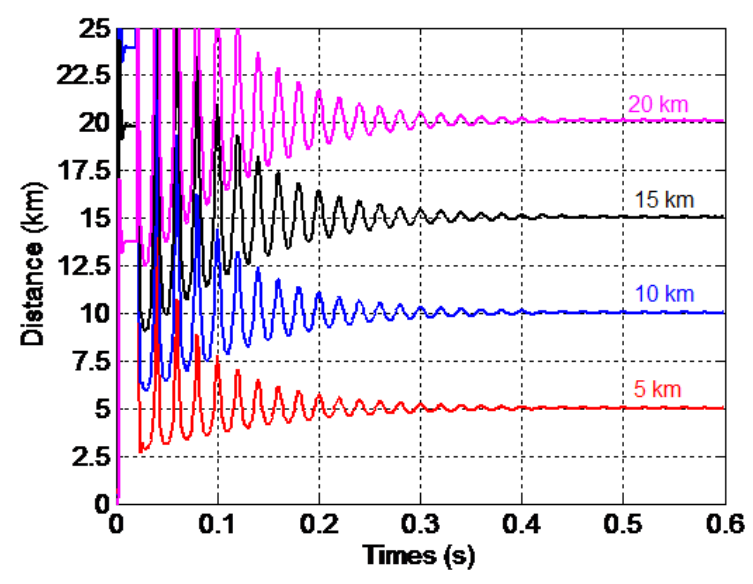

Fig. 12: Fault location as function of time using the reactance for double-phase fault without ground.

From Fig. 11 and Fig. 12 we can see an instability in the response and the distance is not detected rapidly, we can see that the final value 
oscillates around the final value of the supposed fault distance.

Note: the three-phase fault gives us the same results as the double-phase fault with ground.

However, the fault location estimative is affected by many parameters, including fault resistance RF, which may be high for ground faults. In this study we have noted that the maximum value of fault resistance that can be accepted by the proposed technique is $8 \Omega$ for all fault type and at each section.

\section{CONCLUSIONS}

A method of two samples was presented in this paper to detect, classify and localize the fault in the distribution networkwith function of time.The method can be used by numerical relay.The fault detection by the voltage gives a faster response instead of the current. In addition, that faults without ground are classified faster than faults with ground. Concerning the fault locator, for single phase to ground fault, there is stability in the response and the distance is detected rapidly. The distance is determined after $100 \mathrm{~ms}$. For multiphase fault, the fault locator takes some time to the approximate the final value, the response isunstable and the distance is not detected rapidly, but the distance is determined after $400 \mathrm{~ms}$.

\section{References}

[1] Saha, M. M., Das, R., Verho, P., \& Novosel, D. (2002). Review of fault location techniques for distribution systems. Power Systems and Communications Infrastructures for the future, Beijing.

[2] Ganiyu, A. A., Segun, O. S. (2016). An overview of impedance-based fault location techniques in electrical power transmission network. International Journal of Advanced Engineering Research and Applications, 2 (3), 123-130.

[3] Dragomir, M., \& Dragomir, A. (2017). Influence of the Line Parameters in Transmission Line Fault Location. World Academy of Science, Engineering and Technology, International Journal of Electrical, Computer, Energetic, Electronic and Communication Engineering, 11(5), 534-538.

[4] Moravej, Z., Hajhossani, O., \& Pazoki, M. (2017). Fault location in distribution systems with DG based on similarity of fault impedance. Turkish Journal of Electrical Engineering \& Computer Sciences, 25(5), 3854-3867.

[5] Jay, P. K., Harpal T. (2017). Fault location in overhead transmission line without using line parameter. International Journal Of Electrical, Electronics And Data Communication, 5(5), 5-9.

[6] Rui, L., Nan, P., Zhi, Y., \& Zare, F. (2018). A novel single-phase-to-earth fault location method for distribution network based on zero-sequence components distribution characteristics. International Journal of Electrical Power \& Energy Systems, $102,11-22$.

[7] Saini, M., Zin, A. M., Mustafa, M. W., Sultan, A. R., \& Nur, R. (2018). Algorithm for Fault Location and Classification on Parallel Transmission Line using Wavelet based on Clarke's Transformation. International Journal of Electrical and Computer Engineering (IJECE), 8(2), 699-710.

[8] Preeti, G., Mahanty, R. N. (2018). Comparative evaluation of WAVELET and ANN based methods for fault location of transmission lines. International Journal of Pure and Applied Mathematics, 118(19), 25872611.

[9] Guo, M. F., Yang, N. C., \& You, L. X. (2018). Wavelet-transform based early detection method for short-circuit faults in power distribution networks. International Journal of Electrical Power \& Energy Systems, 99, 706-721.

[10] Gururajapathy, S. S., Mokhlis, H., Illias, H. A. B., Abu Bakar, A. H., \& Awalin, L. J. (2018). Fault location in an unbalanced distribution system using support vector classification and regression analysis. IEEJ 
Transactions on Electrical and Electronic Engineering, 13(2), 237-245.

[11] Prasad, A., Edward, J. B., \& Ravi, K. (2017). A review on fault classification methodologies in power transmission systems: Part-I. Journal of Electrical Systems and Information Technology, 5(1), 4860 .

[12] Egeolu, I., Onah, J. (2018). Single phase to ground fault location on $415 \mathrm{v}$ distribution lines using artificial neural network algorithm. International Journal of Innovative Science and Research Technology, 3(1), 371-385.

[13] Seghir, S., Bouthiba, T., Dadda, S., Boukhari, R., \& Bouricha, A. (2018). Fault Location in High Voltage Transmission Lines Using Resistance, Reactance and Impedance. Journal of Advanced Engineering and Computation, 2(2), 78-85.

[14] Filomena, A. D., Salim, R. H., Resener, M., $\&$ Bretas, A. S. (2007, June). Fault resistance influence on faulted power systems with distributed generation. In Proceedings of the seventh international conference on power systems transients, Lyon, France.

[15] Coury, D. V., Oleskovicz, M., \& Souza, S. A. (2011). Genetic algorithms applied to a faster distance protection of transmission lines. Sba: Controle \& Automação Sociedade Brasileira de Automatica, 22(4), 334-344.

\section{About Authors}

Abdelhakim BOURICHA received the M.Sc. degree from University of Science and Technology of Oran city, Algeria in 2016. He is currently a Ph.D. student at the Faculty of Electrical Engineering in the same university. $\mathrm{He}$ is a member of Power System Optimization Laboratory. His research interests include Detection and Location of High Impedance Faults in Medium Voltage Distribution Networks using Neuro-Fuzzy Technique ANFIS and static and dynamic arc fault simulation.
Samira SEGHIR received the M.Sc. degree from University of Science and Technology of Oran city, Algeria in 2015. She is currently a Ph.D. student at the Faculty of Electrical Engineering in the same university. She is a member of Power System Optimization Laboratory. His research interests include fault location in transmission line, dynamic arc fault simulation and numerical relay for transmission line protection.

Rebiha BOUKHARI received the M.Sc. degree from University of Science and Technology of Oran city, Algeria in 2011. She is currently a Ph.D. student at the Faculty of Electrical Engineering in the same university. She is a member of Power System Optimization Laboratory. His research interests include fault location in compensated transmission line and numerical relay in Distance Protection for Series-Compensated Transmission Line using Neuro-Fuzzy Technique ANFIS.

Tahar BOUTHIBA received The Ph.D. degree in Power System in 2004. He is currently a Professor of electrical engineering and a lecturer at the University of Science and Technology of Oran city Algeria. His research interests include computer relaying and control switching using digital techniques and artificial intelligence.

"This is an Open Access article distributed under the terms of the Creative Commons Attribution License, which permits unrestricted use, distribution, and reproduction in any medium, provided the original work is properly cited (CC BY 4.0)." 